\title{
Calidad y Servicios de Prevención de Riesgos Laborales
}

\section{Quality and Occupational Safety and Health Services}

\author{
D. José Luis Castellá López \\ Director Departamento Asistencia Técnica y Normas \\ Instituto Nacional de Seguridad e Higiene en el Trabajo \\ Madrid. España.
}

En el año 2002, transcurridos más de seis años desde la entrada en vigor de la Ley de Prevención de Riesgos Laborales, el Gobierno promovió un doble diálogo, social e interinstitucional, con el objeto de analizar y poder mejorar la situación en dicho ámbito. Como resultado del mismo se concretaron un conjunto de medidas para la reforma del marco normativo de la prevención de riesgos laborales, encaminadas a superar los problemas e insuficiencias respecto de los cuales existía un diagnóstico común, que fueron asumidas en diciembre de 2002 como Acuerdo de la Mesa de Diálogo Social sobre Prevención de Riesgos Laborales y posteriormente refrendadas por la Comisión Nacional de Seguridad y Salud en el Trabajo.

Uno de los principales problemas identificados en el citado diagnóstico fue el hecho, relativamente generalizado, de que el empresario asumía la prevención como una obligación, pero no como una actividad que debía realizarse en la empresa y por la empresa; lo que hacía era "encargar la prevención a los especialistas", es decir, a los Servicios de prevención y, preferentemente, a los Servicios de prevención ajenos. Teniendo en cuenta que el empresario, en general, no disponía de personal propio con conocimientos para juzgar la calidad de las actuaciones concertadas, los factores que a menudo motivaban la elección de uno u otro Servicio eran, por un lado, el precio del servicio y, por otro, el volumen de los informes elaborados por éste, los cuales pasaban a ser considerados por el empresario como la prueba de que cumplía con sus obligaciones preventivas y, por tanto, no iba a ser sancionado. En este contexto no es de extrañar que la Ley 54/2003, de 12 de diciembre, de reforma del marco normativo de la prevención de riesgos laborales, tuviera entre sus objetivos "reforzar la necesidad de integrar la prevención de los riesgos laborales en los sistemas de gestión de las empresas" y "fomentar una auténtica cultura de la prevención de los riesgos en el trabajo, que asegure el cumplimiento efectivo y real de las obligaciones preventivas y proscriba el cumplimiento meramente formal o documental de tales obligaciones".

Posteriormente, en el 2005, en la Mesa de Diálogo Social sobre Prevención de Riesgos Laborales, se acordó impulsar la elaboración de una estrategia española de seguridad y salud en el trabajo, iniciativa que fue refrendada políticamente por el Consejo de Ministros el 22 de abril de 2005. La Estrategia se concibió como instrumento para establecer el marco general de las políticas de prevención de riesgos laborales a corto y, sobre todo, medio y largo plazo, pues abarca el periodo 2007-2012. La Estrategia incluye un diagnóstico de situación en el que se especifica, en relación con la calidad de la prevención, lo siguiente: "La relación entre recursos preventivos propios y ajenos en las empresas, la calidad de las actividades preventivas, la formación de profesionales y trabajadores son cuestiones que pueden y deben mejorarse. Superada la fase de establecimiento de una organización preventiva en las empresas que cumpliera los requisitos legales exigidos, resulta necesario adoptar nuevas medidas y abrir un gran debate entre todos los sujetos implicados sobre cómo perfeccionar la eficacia y la calidad de la prevención de riesgos laborales en nuestro país."

El Objetivo 2 de la Estrategia va específicamente dirigido a mejorar la eficacia y la calidad del sistema de prevención. Entre las líneas de actuación para el desarrollo de este objetivo se encuentran, entre otras, las encaminadas a potenciar los recursos de prevención propios, a favorecer la eficacia y calidad de los Servicios de prevención mancomunados, a reforzar la vigilancia de la salud y a establecer unos criterios de calidad y eficacia exigibles a los Servicios de prevención ajenos basados en "ratios" de medios humanos y materiales de los que deben disponer en función del número de trabajadores, el tiempo de respuesta para los servicios requeridos, los criterios de calidad del servicio y la peligrosidad de las actividades de las empresas con las que tuvieran suscritos conciertos. 
Como no podía ser de otra manera, el proceso de concreción de las distintas medidas mediante las que se desarrollarán esas líneas de actuación ha incluido tanto la cooperación interinstitucional como la consulta con los agentes sociales. El proceso, que comenzó ya hace más de dos años, está a punto de finalizar y contempla la adopción de medidas normativas y no normativas. En relación con las primeras, se prevé la próxima modificación de la Ley de Prevención de Riesgos Laborales y, probablemente durante el 2010, la modificación del Reglamento de los Servicios de Prevención y de la Orden de desarrollo del mismo para incluir, entre otras cosas, los "ratios de medios humanos y materiales".

Entre las medidas no normativas merece destacarse la elaboración, en relación con la actuación de los Servicios de prevención, de unos "criterios de calidad" que puedan ser de utilidad a los propios Servicios, a la hora de gestionar sus actividades, así como a las empresas y sus trabajadores, a la hora de concertar y valorar la calidad de las mismas. Al respecto, debe tenerse en cuenta que la necesidad de reforzar la integración de la prevención en el sistema de gestión en la empresa no va en detrimento del papel que desarrollan los Servicios de prevención; por el contrario, son éstos los que deberán hacer un esfuerzo para facilitar dicha integración. De forma esquemática, puede decirse que los servicios de prevención tienen que realizar una doble función: por un lado, deben gestionar y ejecutar aquellas actividades — tales como la evaluación de los riesgos o la vigilancia de la salud de los trabajadores - que les están reservadas, por su carácter especializado; por otra parte, deben actuar como asesores del empresario en materia de prevención, lo que significa, entre otras cosas, que deben asesorarle para que la prevención quede integrada en la gestión y ejecución de las actividades propias de la empresa. Es de esperar que estos criterios puedan ser aprobados por la Comisión Nacional de Seguridad y Salud en el Trabajo durante el próximo año.

Finalmente, conviene resaltar otras dos medidas, de las previstas en la Estrategia, que facilitarán la mejora de la calidad de la prevención, aunque no estén incluidas en el objetivo específicamente dirigido a tal fin. En primer lugar, se prevé proporcionar asesoramiento público - y ya se ha comenzado a hacerlo- a los empresarios de las empresas de hasta 10 trabajadores para la organización de sus actividades preventivas, impulsando las "autoevaluaciones" y especificando actividades o riesgos que requieren el apoyo técnico especializado; es absolutamente necesario, teniendo en cuenta las dificultades asociadas a los factores de escala, facilitar a estas microempresas el cumplimiento de sus obligaciones en materia de prevención. En segundo lugar, la Estrategia contempla la formulación de un Plan Nacional de Formación en Prevención que, entre otras medidas, prevé la integración de la prevención en la Formación Profesional, la Formación para el Empleo y la Formación Universitaria; con ello se pretende que las personas que vayan desarrollar una determinada actividad profesional estén preparadas para realizarla correctamente, tanto desde el punto de vista productivo como preventivo, lo que sin duda facilitará considerablemente la integración de la prevención en las empresas en que tales personas vayan a trabajar. Se espera poder contar con dicho Plan a mediados del año próximo. 